\title{
Computational Photography: Methods and Applications
}

Rastislav Lukac, Ed., 564 pp., ISBN: 1439817499, CRC Press, Digital Imaging and Computer Vision Series (2010), \$110.53 hardcover.

Reviewed by Hayder Radha, Michigan State University, East Lansing, Michigan

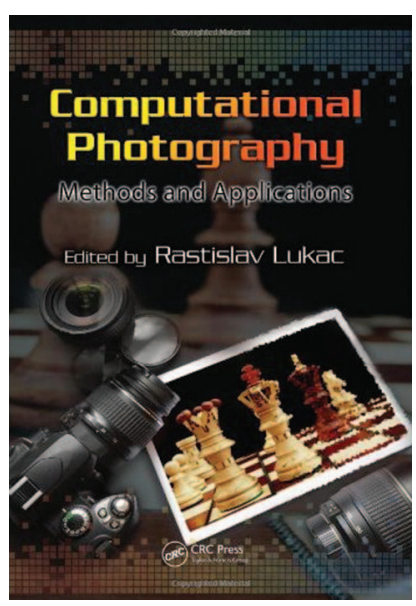

Computational Photography: Methods and Applications offers a rather comprehensive coverage of both the analytical principles and practical algorithms that have been developed for digital photography. Similar to other books that are coordinated by editors rather than written by one or a few authors, this book is a collection of chapters authored by many experts in the broad area of computational photography. A total of 40 authors contributed to 18 chapters resulting in more than 500 pages. The book also includes 32 pages of color images that are replicated from the book chapters; these color images, which form the color insert of the book, are printed on glossy paper for better viewing of the results and performances of the different techniques and methods covered within the book's 18 chapters.

\section{First Impression}

The book begins with a carefully written preface where the editor, Rastislav Lukac, outlines the book chapters, their objectives, and targeted coverage. I believe that Lukac provides a good first impression at the onset of the book by attempting to define the area of computational photography in the first paragraph of the preface. As he highlighted, computational photography "... refers broadly to computational imaging techniques that enhance or extend the capabilities of digital photography" (p. xi). This statement roughly defines the objective of the book as well. Consequently, one can view the book as a collection of (mainly) image-processing methods and algorithms that have been developed to "enhance and extend the capabilities" of modern digital photography. By focusing on this image-processing dimension, the book could arguably be included in any list of recommended reading for a traditional graduate-level image-processing course. Although it could be a stretch, I even found the book as a potential candidate (with some adjustments and updates) to serve as a textbook for a more specialized course in digital photography and imaging. In this latter case, the main missing ingredient relative to a good textbook is a set of homework problems or MATLAB-like projects that are normally found in traditional textbooks. This missing ingredient, however, does not necessarily provide a justifiable ground for criticizing the book in its current version; I do not believe that the book was intended to serve as a textbook. However, I believe that the book has many positive elements that enables it to become a member of a recommended reading list of a graduate-level image-processing course. Despite the clear dominance of "image processing" methods within the book, one would encounter many aspects of computer vision, computer graphics, and machine learning when reading different chapters. Indeed, two chapters (15 and 16) have titles related to machine learning.

\section{Audience}

The clarity in exposition, which begins with the preface, makes the book accessible to a broader audience than experts in the field. Although this is a common claim offered by many editors and book authors, I found the book strikes a reasonable balance between being accessible and analytically deep at the same time. Many parts of this book could be as useful to professionals, who may not have the technical background to appreciate some of the analytical subtleties, as to graduate students, faculty in academia, and other experts, who would probably enjoy some of the deep analysis provided by many chapters. Almost every chapter begins with a high-level description and a big-picture view of the problem at hand. When appropriate, a system-level view is also shown, which reinforces the accessibility of the book to a broader audience than just experts in the field. Each chapter also provides a very decent yet brief overview of major contributions within the particular theme of the chapter. Consequently, the book in its current version also serves as a good pointer to the leading and latest references in digital photography over the past decade and sometimes beyond.

\section{Parts versus Chapters}

Overall, the coverage and the flow of the book chapters are well organized. Although the book chapters are not divided into explicit parts, one can identify a few possible ways for dividing the book chapters into parts that are associated with general themes or areas within computational photography. The editor hints at his own categorization of the book chapters in an implicit way. This categorization can be found in the fifth paragraph of the preface. The editor eludes that the book consists of the following five parts: (a) single capture image fusion; (b) different steps of the imaging pipeline of consumer cameras, including compression, color correction and enhancement, denoising, demosaicking, super-resolution imaging, deblurring, and highdynamic-range imaging; (c) variety of rather loosely coupled topics including bilateral filtering, painterly rendering, shadow detection, and document rectification; (d) machine-learning-based methods; and (e) light-field rendering and dynamic-view synthesis.

\section{Color-Filter-Array Chapters}

After reading the book, I found myself dividing the book into slightly different parts than what was suggested by the editor. For example, I found a relatively clear and common theme 
among five nonsequential chapters.* Chapters $1-3,5$ and 6 all focus on addressing different aspects of the dominant practice of utilizing color filter array (CFA) - based architecture in digital cameras. Within these 5 chapters, the first 2 essentially address the foundation of recovering missing color while maximizing the signal-to-noise ratio of images captured by a single-sensor array. In particular, I found Chap. 1 among the most critical chapters because it describes the foundation of many of the technical and practical building blocks of the popular single-sensor camera architecture. Basic topics such as CFA-based imaging, human visual color response, demosaicking, and other introductory concepts in digital photography are covered. Hence, being the first of 18 chapters, it lays the foundation and describes some of the concepts of the CFA-based camera architecture that are necessary for understanding future chapters. Meanwhile, Chap. 1 also provides a new contribution by advocating the use of a fourth panchromatic color channel (which includes a mix of all three primary colors, and hence, captures the ever important luminance signal) in addition to the traditional three-color CFA channels (chrominance) that are found in the well-known Bayer pattern. The theme of the chapter is the fusion of these different color and luminance channels for the recovery of the original colors while minimizing noise. Hence, the chapter covers many of the foundations of single-sensor cameras in addition to its own contribution related to image fusion of luminance and chrominance information. Consequently, Chap. 1 is the lengthiest, spanning close to 60 pages. Chapter 2 builds upon Chap. 1 by addressing the crucial issue of motion deblurring from a single capture. The chapter describes an interesting approach that exploits the intriguing framework of utilizing a fourth panchromatic channel in addition to the three color channels of the same CFA. This approach is based on the notion of using different exposure times for the different pixel sensors, where the panchromatic channel is sensed (integrated) over a shorter time than the chrominance channels. Both Chaps. 1 and 2 complement each other quite well, which is not surprising given that both were written by common authors, all of whom are leading experts in the area.

Chapter 3 addresses the issue of compressing CFA images in general and Bayer CFA images in particular. The chapter begins by outlining the two major options for incorporating an image codec (encoder and decoder) within a CFA-based camera. The conventional approach would be to perform demosaicking prior to any encoding and decoding operation. In other words, under a traditional approach, standard image compression can be applied directly to a demosaicked image. This chapter describes and advocates the alternative approach of compressing CFA images captured directly by modern digital cameras and storing these compressed CFA images. This alternative approach requires the development of methods that compress CFA images directly, which is the main theme of this chapter.

By continuing within the theme of CFA-related methods, the next chapter in this part of the book is Chap. 5; and hence, we postpone the discussion of Chap. 4 to a later point in this review. Chapter 5 addresses the issue of denoising CFA images. In particular, the chapter describes a principle component analysis (PCA)-based approach for CFA image denoising, and consequently, it provides a brief yet accessible review of PCA, which I believe adds a tangible value not only to the chapter itself but to the book as well. The chapter also reinforces topics intro-

\footnotetext{
*It would have been nice if these chapters, which focus on CFArelated topics, were put in sequence. In particular, by considering the first six chapters 1-6, one may argue that Chapter 4 represents a bit of a disruption of the flow within the context of CFA-based techniques. Nevertheless, this disruption does not take away from the merit of Chapter 4. Furthermore, the placement of Chapter 4 within the first six chapters is consistent with the Editor's own categorization as explained above.
}

duced by other chapters, such as camera imaging fundamentals, including CFA-based camera architecture, demosaicking, traditional camera image-processing pipeline, and last but not least, denoising, which is the main focus of the chapter. Although many of these topics are covered by earlier chapters, I found a slightly repetitive nature of these topics, within the book in general and within the CFA-theme chapters in particular, useful. This brief repetition adds to the accessibility of the book chapters; in addition, it strikes a balance between keeping each chapter self-contained and preventing the book from becoming overly repetitive. Going back to the focus of Chap. 5, and after covering the aforementioned basic topics, the chapter moves on to its description of PCA and noise modeling. Despite its adequate coverage of these two fundamental topics, I believe the chapter could have served the reader better by elaborating further on both topics. For example, I believe that PCA is probably among the most important topics that should be covered thoroughly by any image-processing book. In particular, making a connection with image transforms in general, and with the well-known optimal Karhunen-Loève transform would have been welcome material for many readers. Regarding noise models, the chapter quickly resorts to adopting signal-independent models after a brief admittance that in real life the captured noise by typical image sensors is signal-dependent. Nevertheless, the chapter strikes a reasonable balance by adopting a channel (color)-dependent noise model, yet maintains the traditional signal-independent noise view for tractability and ease of treatment. Other positive aspects of the chapter include its coverage of the denoising procedure where it is based on maximum likelihood estimation (MLE), which is another fundamental topic that should be included in any viable imaging book. One strong aspect of this topic within this chapter is the connection made among MLE, PCA, and the noise model based on a rather pragmatic yet relatively sound approach.

The last chapter in this part of CFA-related material is Chap. 6. This chapter addresses the core topic of demosaicking from an optimization point of view. In particular, it describes a regularization-based demosaicking framework, and hence, this chapter includes several analytically sound concepts that are of interest to theoretically inclined readers. In this context, the chapter touches upon few multirate multidimensional signalprocessing concepts, including periodic sampling and lattices in the spatial and Fourier domains, and integrates these concepts in a rather cohesive analytical framework. Chapter 6 also presents the ill-posed nature of the demosaicking problem and how regularization can help. To that end, the chapter provides a rather compact and relatively accessible treatment of the general topic of regularization for graduate students or other readers who may not be familiar with this topic. Nevertheless, the analytical nature of the material in this chapter makes it inaccessible for a more general audience or those who are not theoretically inclined. This chapter represents an example of why I believe the book can serve as a recommended reading for graduate-level image-processing course.

\section{CFA-less Chapter about Colors}

Chapter 4 addresses the problem of color correction and color constancy. This problem is viewed in this chapter as color restoration and enhancement, which is reflected explicitly by the title of the chapter. Regardless of the problem's name or the chosen title, color constancy is a fundamental issue that is faced by digital photography, and the introduction section of this chapter provides a brief overview about the nature of the problem and its importance. In particular, Chap. 4 provides the traditional explanation for illumination-independent color representation as the de facto objective of color constancy or color-correction algorithms. The theme of the chapter is not color constancy per say, but rather on how to approach this problem in the compressed domain. Given 
that the DCT is the dominant transform for leading imaging compression methods, the chapter provides a brief overview of DCT fundamentals. The chapter provides a very extensive coverage of simulation results with plenty of examples. A color-enthusiast reader, however, might be potentially disappointed when reading the simulation results. Both the unfortunate exclusion and the print quality of some of the key color images (from/within the color insert of the book) may cause a bit of distress for color fanatics. Although a few important images, which are shown as gray-level images within the corresponding book chapters, have unfortunately been excluded from the designated color pages, I believe all images of Chap. 4 dealing with color correction should have been replicated and included in the insert of color pages. More importantly, I believe that the quality of some of the images included in the color insert does not serve the reader much in appreciating the subtle differences among the different colorcorrection methods. Many of the images from Chap. 4 included within the color insert are a bit dark with little or no visible color information. Nevertheless, a couple of color examples do provide a visual confidence about the chapter's theme and contribution.

\section{Super-Resolution with Super Rigor}

For an academic reader, Chap. 7 is arguably the best chapter in this book. It provides an excellent exposition of the important area of super-resolution (SR). As a start, the topic of the chapter is among the most interesting and important areas within computational photography. More importantly, the way the chapter is written and the way the topic is covered makes Chap. 7 worthy material to browse, read, and even study for anyone who is interested in the area of computational photography. One of the reasons why an academic person (like me) would enjoy this chapter is that it covers a crucial set of fundamental topics in a comprehensive and well-written way, with the appropriate level of analytical depth. Another reason for the success of the chapter is its avoidance of presenting a new contribution. As highlighted by the author, the chapter "aims to serve as an introductory material for SR imaging, and also provide a comprehensive review of SR methods" (p. 177). The chapter brings together topics such as aliasing, blurring, and noise models in a cohesive way, and integrates them quite clearly into the SR imaging inverse problem. A multitude of core and popular approaches for SR are covered. These topics range from frequency domain methods to joint interpolation and deconvolution, least square estimation, and Bayesian estimation. More recent and advanced approaches are also covered, such as projection onto convex sets and training-based SR imaging. The chapter even goes further by addressing another set of important topics and how they impact the problem of SR. These topics include image registration, regularization and parameter estimation, blur modeling, and other advanced imaging model issues and problems. The chapter concludes its excellent exposition by addressing the CFA architecture, and hence, it positions itself as self-contained as any other chapter in the book. In short, Chap. 7 is must-read material for a broad range of readers in the area.

\section{Traditional Application with More Rigor}

Despite a difference in application, there is a common formulation between the topics of Chap. 7 (super-resolution) and Chap. 8 (image deblurring). Hence, Chap. 8 presents a general degradation model that captures image blurring. This model includes both the traditional convolution by a shift-invariant point spread function and an additive noise model. Both of these degradation elements can be found in the SR formulation of Chap. 7; however, Chap. 7 addresses further issues such as aliasing (due to subsampling), and many other fundamental and advanced topics as outlined above. Chapter 8 subsequently addresses the problem of deblurring using a classical deconvolution approach based on a regularization framework. Other image-deblurring aspects, which do not rely on deconvolution, are also covered. Overall, Chap. 8 covers one of the most important and traditional topics in digital photography, namely deblurring, yet it is rather brief in its treatment, especially when compared to Chap. 7. Meanwhile, I found the two chapters complement each other in the sense of addressing two important problems in digital photography, yet outlining similar analytical formulations with some welcomed rigor to address these problems.

\section{High-Dynamic-Range Imaging}

The next two chapters of the book, Chaps. 9 and 10, cover the increasingly popular tool of high-dynamic-range (HDR) imaging. In particular, Chap. 9 provides a very good introductory overview of HDR imaging. The chapter covers both the capturing (composition) and display (tone mapping) aspects of the HDR problem in a way accessible to a broad set of readers. Similar to other chapters (e.g., the one on super-resolution and the chapter on deblurring), Chap. 9 addresses the problem of HDR using multiple exposures. One positive aspect of the chapter is that it covers the HDR problem using a rather generic luminance-chrominance space. Consequently, the chapter begins with a very easy-toread review of popular color spaces, especially those that are relevant to the HDR problem (e.g., opponent, YUV, and HSV color spaces). This type of coverage makes the chapter selfcontained and comprehensive in terms of being an introductory tutorial to the important topic of HDR imaging. For the capturing (composition) side of HDR, the chapter addresses key algorithms for monochrome and luminance-chrominance-based HDR composition methods. This is followed by a parallel coverage of monochromatic and luminance-chrominance display (tone mapping) methods. The chapter also includes a brief overview of hardware-based HDR solutions. Plenty of illustrative examples are included as well, many of them can be found in the color insert pages of the book. Chapter 10 continues the topic of HDR but adds a new and practical dimension to the problem, specifically the issue of achieving HDR imaging under motion and dynamic scenes. To be self-contained, the chapter begins with a very brief overview of the topic of HDR and the more specific issue of HDR imaging from multiple exposures. This overview intersects with the material from Chap. 9 and may seem redundant; however, it reinforces some of the key concepts. One arguably negative issue, which is probably unavoidable in an editorial book, is a minor inconsistency in notations that are used in Chaps. 9 and 10. Nevertheless, I found both chapters provide a very good overview and detailed discussion of the increasingly important topic of HDR, which in my judgment, represents one of the strong and positive aspects of the book.

\section{New and Emerging Problems and Applications}

The remainder of the book covers a rather interesting and sometimes intriguing set of applications and related imaging problems. Starting with Chap. 11, and arguably with the exception of Chap. 13, the book takes the reader on a tour of new problems ranging from the practical, such as surveillance, to more artistic, such as painting and colorization.

Chapter 11 provides an interesting overview and describes a new approach for shadow detection. The chapter outlines a very easy-to-read taxonomy of (a) the broad set of applications that benefit from or need shadow detection, and (b) the different approaches and algorithms used for shadow detection. In particular, the chapter focuses on region-based parametric modeling of the shadow detection problem for surveillance video applications. The chapter categorizes the problem of shadow detection as nonparametric (illumination independent) and parametric; then it divides parametric approaches into local and global ones; finally, it highlights its focus on a global parametric framework that it 
introduces. The description of this framework is approached by the chapter in a very clear way (at least from a high level). This clarity is accomplished by distinguishing and addressing the two issues of shadow detection: statistical feature modeling and color space analysis. As the chapter author explains, this strategy in exposition was done by design, not by accident. Despite the extensive interaction between these two issues/aspects (statistical feature modeling and color space analysis) of the shadow-detection problem, treating both subjects in separate sections emphasizes both the importance of, and the distinction between, these two issues. Both benchmark sequences and real-life surveillance video were used to present many illustrative results that attempt to validate the introduced framework in this chapter. Despite the potentially perceived narrow application space (surveillance) and described framework (parametric-based global) outlined in this chapter, I found it a very helpful reference for this increasingly important area for computational photography.

Chapter 12 covers an intriguing and emerging digital imaging application for scanning using digital cameras as opposed to employing traditional flatbed scanners. This application requires what is known as document dewarping or document rectification, which is the focus of this chapter. As highlighted by the chapter, document dewarping/rectification is needed for addressing perspective and geometric distortions that result from using regular digital cameras when capturing documents. After a very brief overview of different approaches for document rectification, the chapter makes arguments about the disadvantages of these approaches in preparation for motivating the authors' proposed methods that are covered in this chapter. This aspect of the chapter, although consistent with its objective of presenting the authors' own approaches, has a bit of a negative tone, even for an editorial book. ${ }^{\dagger}$ The chapter proceeds by focusing on two major digital camera-driven methods for image rectification: (1) a stereo-based approach for document rectification, and (2) a single-image-based method for figure rectification. Both approaches are covered with sufficient analytical depth and many illustrative examples. As anyone in the area may expect, the stereo-based method is based on explicit three-dimensional (3D) reconstruction. This approach is naturally more versatile than the second (single-image-based) method in terms of its ability to work in conjunction with optical character recognition. The focus of the single-view-based approach is on rectifying a figure within a scanned document. Although the chapter makes a clear case for the viability and potential of camera-driven document scanning, this chapter could have served a more comprehensive purpose by surveying other techniques, and more importantly, by addressing related key imaging issues. To the authors' credit, they admit, albeit late in the conclusion section, that other important and related issues to document rectification, such as uneven illumination and motion blur, were not addressed by the chapter. Despite these shortcomings, the chapter does bring this very interesting and relatively new topic to the reader with a good list of references and illustrative figures.

\section{Old Filter with a New Name, Improved Design, and More Rigor}

Chapter 13 provides an overview of the theory and applications of bilateral filters. Before proceeding with my review of the chapter, it is worth noting that I found the chapter placement within this last part of the book (Chaps. 11-18) a bit odd. This last part of the book apparently focuses on new and emerging

†Naturally, this is a common struggle for anyone writing a book chapter: how much of it should be a fair survey, and how much of it should be a coverage of the authors' own and preferred methods. Therefore, this comment is not intended as a criticism of this particular chapter, but a rather an observation related to all chapters that emphasize the authors' contributions versus surveying related work. applications, yet Chap. 13 addresses a specific class of filters. Probably the rational for placing Chap. 13 within this part of the book is the following: bilateral filters, which take into consideration both the spatial distance and intensity distance of neighboring pixels with respect to the pixel under consideration, have found many compelling applications due to their robustness in preserving critical image details, such as edges, while achieving superior smoothing results. The chapter provides a very good overview of the theoretical aspects and applications of bilateral filters, yet I believe that this topic has much deeper roots than the coverage provided by the chapter. For example, from the early and mid1980s, one active area of research was order-statistic filters and related design (such as alpha-trimmed and even special cases of median filters). I believe that the chapter would have served the reader more by providing some mention of these related topics. Nevertheless, the chapter provides an excellent outline of the utility of bilateral filters to a broad range of important areas such as denoising, tone mapping, artifact reduction (e.g., due to compression), and other related applications. Fast algorithms for bilateral filters are also covered.

\section{Back to the Intriguing Application}

Chapter 14 covers arguably one of the most intriguing applications of modern digital photography called painterly rendering. In its most simplistic form, this area addresses the basic notion of converting natural images taken by digital cameras to pieces of visual art. The chapter captivates the reader from the first paragraph by explaining and motivating this new area by providing examples of fascinating interactions between art, psychology, and digital imaging. The chapter briefly and quickly outlines a relatively long list of references related to this emerging area, and then it explains its focus, that is unsupervised painterly rendering, which automatically converts any natural image into what is known as a "painterly image." The chapter explores two sets of algorithms: (1) ones that simulate putting paint on paper or canvas, and (2) other algorithms for abstracting from the classical tools used by artists over centuries until our modern time. The chapter focuses on describing algorithms and related models for the simulation of brush strokes. Furthermore, the chapter addresses the visual properties that distinguish an artistic work, such as classical painting, from a natural image taken by a camera. This later topic also covers adding synthetic texture based on the theory of glass patterns. The chapter includes a very good number of beautiful images that are included in the color insert. In summary, for those who enjoy painting and visual art, this is probably the most enjoyable chapter in the book!

Chapter 15 arguably continues the artistic theme of Chap. 14, yet it covers a completely different imaging application. In particular, Chap. 15 addresses the ill-posed problem of automatic colorization of grayscale images. Automatic is a key word in this context. The introductory part of the chapter makes a convincing argument about the limitation of manual colorization methods when applied to grayscale visual content, and hence, it provides a good motivation for approaching the problem using learning techniques that are developed within the area of machine learning (ML). More importantly, the chapter outlines well-established ML methods for fully automatic image colorization, which can be applied to this problem without any user intervention. The chapter also argues about adopting global methods (as opposed to local ones) to achieve this rather challenging objective of fully automating the colorization process. The chapter formulates image colorization as a prediction problem; and it outlines nonparametric methods, namely the Parzen window and the well-known support vector machine (SVM) methods, for tackling this problem. The chapter also covers graph-cut algorithms, which the author argues provide the best results due in part to their ability to combine local predictions with spatial coherency functions. 
Overall, I found the chapter interesting (considering both the application and the ML tools), informative (in terms of its coverage of well-established ML frameworks and concepts), and relatively accessible, especially for those who might have some background in $\mathrm{ML}$ or signal processing. Otherwise, some parts of the chapter might be a bit challenging to follow for readers who are not very familiar with basic ML concepts. Nevertheless, I believe the chapter provides a very decent coverage of these ML concepts, such as SVMs, or at least it provides sufficient pointers to them for those who are interested in learning more. In summary, even for those who might not be familiar with ML, they would leave the chapter familiar with some of its most common jargons, and with a sense of the viability and applicability of ML for automatic image colorization.

Chapter 16 has commonalities with both Chaps. 14 and 15: it arguably maintains the book's artistic theme, which begins in Chap. 14, and it covers some ML approaches, which represent a major theme of Chap. 16. The focus of Chap. 16 is face beautification, an application that is both intriguing and controversial! The tools covered in the chapter for this application are drawn from the rich area of machine learning. Although most humans will not argue against the viability of face beautification as an application, the chapter begins with strong motivating statements, such as the human face being the "most frequently photographed object on earth" (p. 421). The chapter also provides a brief window into some of the philosophical, psychological, and artistic arguments for face beautification. One of the salient points highlighted in this chapter is the observation that many studies have shown a consistent perception and agreement among many cultures regarding the human perception of face attractiveness. As the author stated, "... people share a common taste for facial attractiveness" (p. 422). I believe that this statement, which is cleverly repeated and strategically placed in multiple occasions within the first few pages of this chapter, provides a convincing reason for the interested reader to continue reading or at least glancing at this chapter. After the well-written introductory part, the chapter covers supervised learning methods for building a facial beauty model. This is followed by descriptions of both an optimization approach and a heuristic method for face beautification. One of the excellent aspects of the chapter is its coverage of some of the details related to the whole process of this application. This coverage is initially presented well by showing a comprehensive overall system diagram of the different functions and processes needed to develop a face beautification application. Then, each of the individual components of the system is covered, usually within a designated section of the chapter. The chapter could have easily stopped its coverage of the topic after the first four sections; however, the author continued by presenting details regarding methods for locating face features and for identifying canonical points on frontal images of human faces. It also includes coverage of how to perform the required image warping that ensures unmistakable resemblance to the original image while achieving the sought after beautification. The chapter even includes a brief appendix to elaborate further on one of the key ML approaches presented in the chapter, called support vector regression.

\section{Light Field Rendering}

The last two chapters, 17 and 18, cover the area of light field imaging. Although both chapters provide some form of an introduction to the area of light field rendering, Chap. 18 outlines a more comprehensive overview (or a survey) of the area's major contributions. Hence, the book reader might have been served better if the order of these two chapters was reversed. The focus of Chap. 17 is on addressing two major issues with traditional light field rending methods: a. trading off spatial resolution versus angular resolution and b. what the authors call "common photometric distortion and aliasing" (p. 446). As a consequence, the chapter is outlined and written as a journal paper where the authors "propose" a new framework that includes a hardware solution, programmable aperture, and post-processing algorithms for removing distortions associated with light field rendering and for generating view-dependent depth maps for view interpolation. Overall, Chap. 17 is well-written and should be accessible to a relatively broad audience despite its shortcoming in terms of providing an overview of the area and related work. Nevertheless, this shortcoming of Chap. 17 is compensated for in Chap. 18 where a much more comprehensive survey of related work in the area is provided. Meanwhile, and as part of its outline of the different contributions in the area, Chap. 18 provides an indepth description of the authors' contributions in dynamic view synthesis using areas of cameras. One of the positive aspects of Chap. 18 is that it provides a very good perspective of the area of light field modeling and rendering, and how it fits within the larger context of image-based modeling and rendering. Furthermore, in its overview of the different contributions, which tend to be mostly chronological, the chapter moves from the most general five-dimensional plenoptic function model toward fourdimensional, 3D, and even two-dimensional light field imaging models. This flow of presentation, I believe, can be very helpful for readers who are looking for guidance on how these different areas relate to each other and how they fit within the larger contexts of light field modeling and image-based rendering.

\section{The Last Word}

Overall, the book presents very well-balanced material for a broad audience in digital and computational photography. The book provides an arguably excellent coverage of analytical tools (from traditional image processing to computer vision and machine learning), traditional applications (such as demosaicking, denoising, deblurring, and super-resolution), to emerging and intriguing applications (such as painterly rendering and automatic colorization). It is always unknown to a reader of an anthology how the editor chooses the particular experts who end up writing the chapters of such a book, especially when there are many competing research groups covering a particular theme of a chapter. For this book, I believe that the editor has made firstrate choices, and as a consequence, I believe that the chosen authors with their contributions have served their editor and their readers quite well.

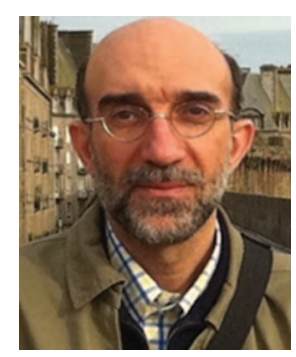

Hayder Radha received $\mathrm{PhM}$ and $\mathrm{PhD}$ degrees from Columbia University in 1991 and 1993, an MS degree from Purdue University in 1986, and a BS degree (with honors) from Michigan State University (MSU) in 1984, all in electrical engineering. Currently, he is a professor of electrical and computer engineering at MSU and the director of the Wireless and Video Communications Laboratory. He was with Philips Research from 1996 to 2000, where he worked as a consulting scientist and fellow in the Video Communications Research Department. He was a member of the technical staff (MTS) and then a distinguished MTS at Bell Laboratories where he worked between 1986 and 1996 in the areas of digital communications, image processing, and broadband multimedia. $\mathrm{He}$ is a Fellow of the IEEE, and his current research areas include multimedia communications and coding, image processing, compressed sensing, social networks, sensor networks, and network coding. He has authored more than 150 peer-reviewed papers and 30 U.S. patents in these areas. 